\title{
Radar Measurement of Human Polarimetric Micro-Doppler
}

\author{
David Tahmoush and Jerry Silvious \\ U.S. Army Research Lab, Adelphi, MD 20783, USA \\ Correspondence should be addressed to David Tahmoush; david.tahmoush@us.army.mil
}

Received 24 May 2013; Accepted 4 September 2013

Academic Editor: Sandra Costanzo

Copyright (C) 2013 D. Tahmoush and J. Silvious. This is an open access article distributed under the Creative Commons Attribution License, which permits unrestricted use, distribution, and reproduction in any medium, provided the original work is properly cited.

\begin{abstract}
We use polarimetric micro-Doppler for the detection of arm motion, especially for the classification of whether someone has their arms swinging and is thus unloaded. The arm is often bent at the elbow, providing a surface somewhat similar to a dihedral. This is distinct from the more planar surfaces of the body which allows us to isolate the signals of the arm (and knee). The dihedral produces a double bounce that can be seen in polarimetric radar data by measuring the phase difference between HH and VV. This measurement can then be used to determine whether the subject is unloaded.
\end{abstract}

\section{Introduction}

Detailed radar processing can reveal many characteristics of human motions and of the human body, including gait characteristics. Micro-Doppler signals refer to Doppler scattering returns produced by the motions of the target other than gross translation. Parts of the human body do not move with constant radial velocity; some of the small micro-Doppler signatures are periodic, and therefore analysis techniques can be used to obtain more characteristics [1,2]. Micro-Doppler gives rise to many detailed radar image features in addition to those associated with the bulk target motions. Modulations of the radar return from arms, legs, and even body sway are being investigated by researchers [3-5]. There are also some tutorials on micro-Doppler phenomena $[2,6,7]$.

The Doppler information measured by a radar arises from target motions. The equation for computing the nonrelativistic Doppler frequency shift, $F_{d}$, of a simple point scatterer moving with speed $v$ with respect to a stationary transmitter is

$$
F_{d}=F_{t} \frac{2 v}{c} \cos \theta \cos \phi
$$

where $F_{t}$ is the frequency of the transmitted signal, $\theta$ is the angle between the subject's velocity and the beam of the radar in the ground plane, and $\phi$ is the elevation angle between the subject's velocity and the radar beam. This assumes that the radar itself is stationary. Targets can be considered as collections of simple scatterers, though this is a rough approximation. The micromotion of the scatterers around the center frequency creates a micro-Doppler model that varies with time. Several micro-Doppler models have been developed which characterize and attempt to predict the human micro-Doppler response [8-10] using animated collections of simple scatterers as the foundation.

A short-time FT (STFT) is one way to explore the slow time-dependent behaviour of the Doppler spectrum by doing a Fourier transform over a small window in time, then sliding the window [11]. This avoids the loss of time information that occurs when applying a Fourier transform. The continuous form of the STFT is

$$
\operatorname{STFT}(x(t))=X(\tau, \omega)=\int_{-\infty}^{\infty} x(t) w(t-\tau) e^{-j w t} d t,
$$

where $w(t)$ is the window function. Because human microDoppler varies slowly with time, we employ STFTs of the IQ radar data. The length of time used in the STFT is called coherent processing window, and this determines the resolution in Doppler frequency that can be measured. This can partially be overcome by superresolving methods. The spectrogram is the square modulus of the STFT and is then

$$
\operatorname{spectrogram}(\tau, \omega)=10 \log _{10}|X(\tau, \omega)|^{2}
$$

which is often used to display micro-Doppler data in decibels. Much of the analysis in this report makes use of spectrograms for the display of micro-Doppler phenomenology. 
The extraction of micro-Doppler features is typically performed in the joint time-frequency domain. Chirplet techniques [12] as well as linear FM basis decomposition [13] can be used to perform feature extraction. Independent component analysis (ICA) can be used to extract independent basis functions from the spectrogram to be used as features in a classifier [14]. Micro-Doppler signatures have been suggested as a biometric [15], and micro-Doppler features have been used in classification algorithms [15-18]. MicroDoppler signatures have been extracted through a brick wall [19]. Fully polarimetric human radar signatures at different approach angles with respect to the radar have been collected [20]. Automatic target classification has also been done on data including multiple humans, wheeled vehicles, tracked vehicles, clutter, and animal classes [21]. Micro-Doppler phenomena have been investigated in frequencies as low as UHF [22]. A $77 \mathrm{GHz}$ radar was used to observe micro-Doppler signatures of human gait to recognize multiple persons and attempt to identify whether the person is swinging their arms [23]. An ultrawide band (UWB) impulse radar was used to provide both high resolution range profiles and high resolution Doppler spectrogram, which helps to extract detailed micro-Doppler signatures like swinging arms [24]. The detailed signatures are used to recognize human activities, such as marching, walking, one-arm swinging, or twoarm swinging. A combination of micro-Doppler signatures with microrange features was also proposed [25].

\section{Models}

To understand the micro-Doppler presented by moving humans, a model was built using the human motion as a collection of simple scatterers. Several other micro-Doppler models have been developed that characterise and attempt to predict the human micro-Doppler response [8-10]. We use research on human gait to model the expected Doppler shifts measured over time by a radar system. We started with the measurements made on twenty men and twenty women whose ages ranged from 20 to 38 years with an average age of 26 years and had their motions captured on video and extracted then their characteristics analyzed [26]. The resulting motion information was extracted, and then animated. We took the animated gait and extracted the micro-Doppler velocities that would be created by differentiating the motions using a point-scatterer model for each separate part.

We neglected obscuration for these simulations because they were limited to frontal view, and we used a metallic skin approximation to simplify the calculations by neglecting the skin depth. The simulated micro-Doppler motions for different body parts are compared to measured data. These are calculated from the model and are calculated and measured at $17 \mathrm{GHz}$. The scaling for the images was set in order to simplify the comparison of images to demonstrate the variability of the human gait as viewed by the radar. The stride rate is also held fixed to simplify comparisons. We also do not simulate noise in the models. Highly accurate mesh-modeled simulations of the human micro-Doppler signature have also been done [27] at lower frequencies where the metallic skin approximation does not apply.

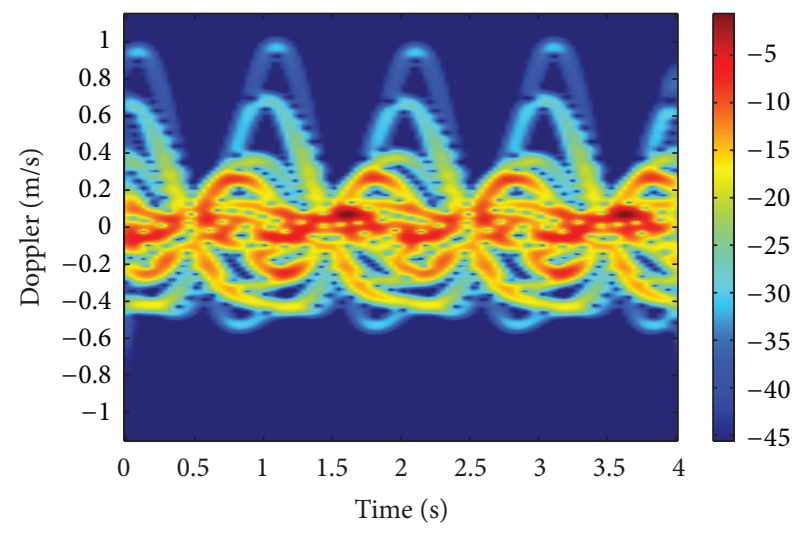

FIgURE 1: Simulated micro-Doppler for a man walking on a treadmill in the radial direction toward the radar at $17 \mathrm{GHz}$.

The usefulness of the simulations is in the ability to understand the measured effects seen in different radar scenarios. They also provide the ability to create a simulated database to test algorithms and approaches for feature extraction and classification. There is not a perfect agreement between simulated and actual measurements, but much of the qualitative signature aspects are in the simulations. The torso line, foot swing, and arm motions are all contained in the simulation. This compares well to other micro-Doppler simulations $[9,15]$. We then added radar cross sections (RCS) to the simulations by modeling the human body as a set of elliptical shapes and calculated the resulting spectrogram as shown in Figure 1.

Now that we have a qualitatively validated model, we are going to use it to analyze the results of polarimetric human micro-Doppler measurements.

\section{Polarimetric Micro-Doppler}

Fully polarimetric radar data can potentially improve the separability of different parts of human motion. One of the techniques to determine whether someone is carrying a load is looking at their arm motion [28]. The arm is often bent at the elbow, providing a surface similar to a dihedral which could create a double bounce. This is distinct from the more planar surfaces of the body and allows us to separate the signals of the arm (and knee) motion from the rest of the body. The double bounce can be measured in polarimetric radar data by measuring the phase difference between $\mathrm{HH}$ and VV [29]. Measurements of humans were taken at an outdoor radar test range with realistic but low levels of clutter at Ka band. Further descriptions of the radar system, test range, clutter, and other parameters are available in [20].

A standard spectrogram model of the micro-Doppler of a human walking motion is shown in Figure 1. The dominant phases for a walking human similar to Figure 1 are measured and shown in Figure 2. Note that the characteristic foot swing is still somewhat discernible. There is a significant number of signals with a high ( $>150$ or $<-150$ degrees) phase shift in the torso-line area of the motion. Figure 3 shows the dismount with the simulated motion of elbows superimposed, which 


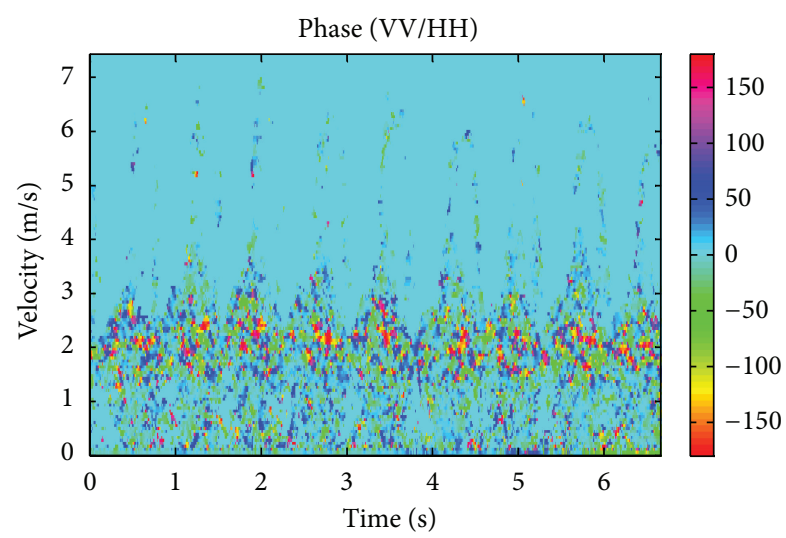

FIGURE 2: Polarimetric measurement of human walking toward the radar. The red (and yellow) double-bounce areas correspond to the elbow motion, while the blue (and green) single bounce is the rest of the body.

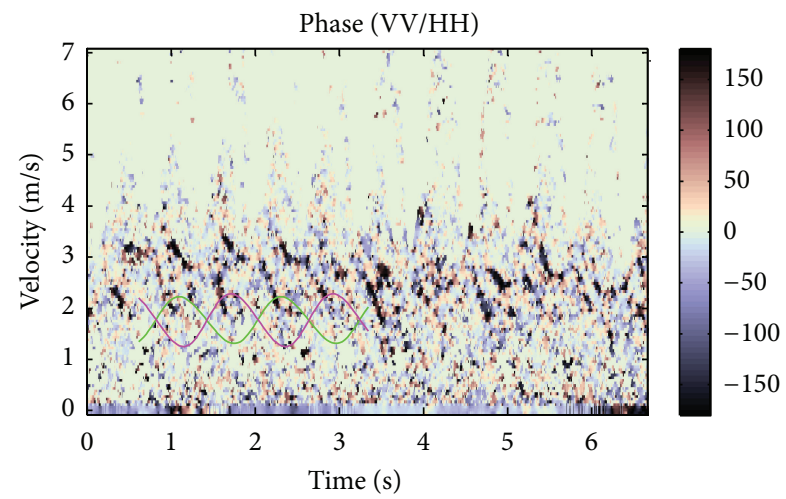

FIgURE 3: Polarimetric measurement of an unloaded human walking toward the radar at Ka band from Figure 2 with the simulated elbow movement superimposed as red and green lines roughly corresponding to the areas of significant phase offset from zero.

matched reasonably well the measured areas of high phase offset from zero. Note that the elbow maxima are simulated to be slightly before the foot maxima, as shown in Figure 1 .

The repeatability of the measurement is an issue. Two measurements were made of an individual walking away from the radar, with the knees being highlighted instead of the elbows. This is shown in Figures 4 and 5 where a measurement of knee polarimetric response is repeated and the simulated motion is superimposed. An additional issue is the effect of angle on the measurement response. Polarimetric microDoppler with a double bounce was only clearly detectible at small offsets from the radial direction in azimuth or elevation. The arm is smaller in RCS than the torso or leg, so when both signals are present, the smaller arms signal is not as distinguishable. This meant that the angles where it is easy to view the double bounce returns from the elbow and knee are when the arm is readily discernible from other signals.

When a dismount is loaded, the arm motion is restricted so there is less opportunity to easily observe the double bounce. Figure 6 shows a loaded dismount which can be compared to Figure 2. It is clear from the differences in

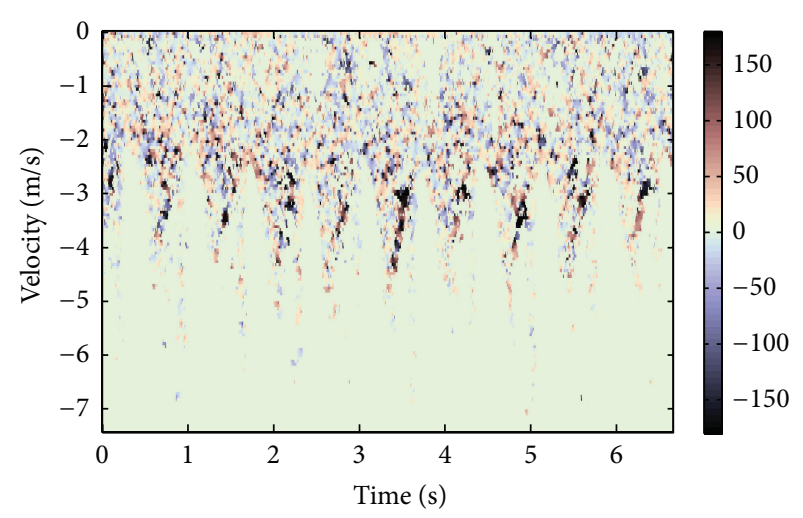

FIgURE 4: Polarimetric measurement of a human walking away from the radar at Ka band. The black double-bounce areas correspond to the elbow motion, while the white and grey single-bounce area is the rest of the body.

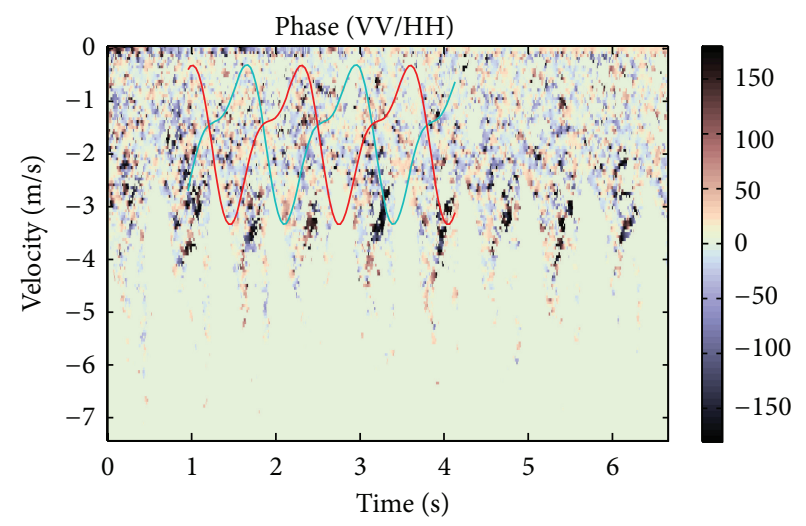

FIGURE 5: Repeated polarimetric measurement of a human walking away from the radar at Ka band. The black double-bounce areas correspond to the elbow motion, while the white and grey singlebounce area is the rest of the body. The simulated motions of the knee are superimposed on the image, showing a rough correspondence to areas of high doublebounce.

Figures 2 and 6 that there is potential in utilizing polarimetric radar in classifying dismounts as having their arms loaded or freely swinging. The simulated arm motion is overlaid in Figure 3. The polarimetric measurement of human walking toward the radar at Ka band while carrying a load in Figure 6 shows that the red double-bounce areas corresponding to the elbow motion are severely diminished because the dismount can no longer swing the arms freely. Dismounts carrying different objects can appear to have similar motion, though this was not true with the most common implements [28]. This study is not definitive because of the small amount of data with limited statistics, but the potential to utilize polarimetry to distinguish loaded versus unloaded dismounts is shown.

\section{Conclusions}

Extensive fully polarimetric outdoor radar human microDoppler walking signatures have been collected and analyzed. Micro-Doppler simulations were created and 


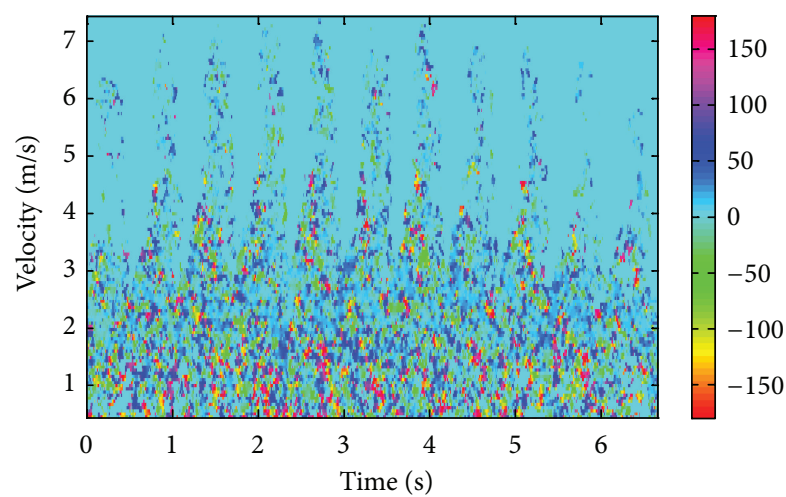

Figure 6: Polarimetric measurement of a loaded human walking toward the radar at Ka band. The red (and yellow) double-bounce areas correspond to the elbow motion, while the blue (and green) single bounce is the rest of the body. Comparison with Figure 2 shows a reduction in the arm motion.

qualitatively validated against the measured data, allowing the use of the simulations to analyze the polarimetric micro-Doppler returns. The motion of humans is shown to have measurable double-bounce returns from both the elbows and knees similar to a dihedral. These returns vary when the human is loaded versus unloaded, primarily in the double-bounce return from the freely swinging arms. Thus, the extraction of polarimetric micro-Doppler features from radar data has been shown to be feasible and potentially useful.

We discovered several important characteristics that should be considered for the measurement of human microDoppler. First, since the arm motion is important for the determination of whether a person could be loaded, polarimetric measurements can isolate and highlight the arm motion for a classification as being loaded or unloaded. Second, the azimuthal angle of the motion is a critical parameter to consider in ground-based systems. For chokepoint observations where the direction of motion is more controlled, polarimetric radar could be used to determine who is unloaded and who should be checked.

\section{References}

[1] L. Cohen, Time-Frequency Analysis, Prentice-Hall, Englewood Cliffs, NJ, USA, 1995.

[2] V. C. Chen and H. Ling, Time-Frequency Transforms for Radar Imaging and Signal Analysis, Artech House, Norwood, Mass, USA, 2002.

[3] S. Z. Gürbüz, W. L. Melvin, and D. B. Williams, in Proceedings of the SPIE Signal Processing, Sensor Fusion, and Target Recognition XVI, vol. 6567, 2006.

[4] G. Greneker, "Very low cost stand-off suicide bomber detection system using human gait analysis to screen potential bomb carrying individuals," in Proceedings of the Radar Sensor Technology IX, vol. 5788, pp. 46-56, Orlando, Fla, USA, March 2005.

[5] J. L. Geisheimer, W. S. Marshall, and E. Greneker, "A continuous-wave (CW) radar for gait analysis," in Proceedings of the 35th Asilomar Conference on Signals, Systems and Computers, pp. 834-838, November 2001.
[6] V. C. Chen and R. Lipps, "Time-frequency signatures of microDoppler phenomenon for feature extraction," in Proceedings of the Wavelet Applications VII, pp. 220-226, April 2000.

[7] V. C. Chen, F. Li, S.-S. Ho, and H. Wechsler, "Micro-doppler effect in radar: phenomenon, model, and simulation study," IEEE Transactions on Aerospace and Electronic Systems, vol. 42, no. 1, pp. 2-21, 2006.

[8] D. Tahmoush and J. Silvious, "Radar microdoppler for security applications: modeling men versus women," in Proceedings of the IEEE International Symposium on Antennas and Propagation and USNC/URSI National Radio Science Meeting (APSURSI '09), Charleston, SC, USA, June 2009.

[9] P. Van Dorp and F. C. A. Groen, "Human walking estimation with radar," IEE Proceedings: Radar, Sonar and Navigation, vol. 150, no. 5, pp. 356-366, 2003.

[10] D. Tahmoush and J. Silvious, "Angle, elevation, PRF, and illumination in radar microdoppler for security applications," in Proceedings of the IEEE International Symposium on Antennas and Propagation and USNC/URSI National Radio Science Meeting (APSURSI '09), Charleston, SC, USA, June 2009.

[11] A. V. Oppenheim and R. W. Schafer, Discrete-Time Signal Processing, Prentice Hall, Englewood Cliffs, NJ, USA, 1989.

[12] J. Li and H. Ling, "Application of adaptive chirplet representation for ISAR feature extraction from targets with rotating parts," IEE Proceedings: Radar, Sonar and Navigation, vol. 150, no. 4, pp. 284-291, 2003.

[13] P. Setlur, M. Amin, and F. Ahmad, "Analysis of micro-doppler signals using linear FM basis decomposition," in Proceedings of the Radar Sensor Technology X, Orlando, Fla, USA, April 2006.

[14] V. C. Chen, "Spatial and temporal independent component analysis of micro-Doppler features," in Proceedings of the IEEE Radar Conference, pp. 348-353, May 2005.

[15] J. L. Geisheimer, E. F. Greneker, and W. S. Marshall, "A highresolution doppler model of human gait," in Proceedings of the Radar Sensor Technology and data Visualisation, pp. 8-18, Orlando, Fla, USA, April 2002.

[16] Y. Yang, J. Lei, W. Zhang, and C. Lu, "Target classification and pattern recognition using micro-doppler radar signatures," in Proceedings of the 7th ACIS International Conference on Software Engineering, Artificial Intelligence, Networking, and Parallel/Distributed Computing, SNPD 2006, including Second ACIS International Workshop on Self-Assembling Wireless Networks (SAWN '06), pp. 213-217, Las Vegas, Nev, USA, June 2006.

[17] P. G. Kealey and M. Jahangir, "Advances in Doppler recognition for ground moving target indication," in Proceedings of the Automatic Target Recognition XVI, Orlando, Fla, USA, April 2006.

[18] M. Otero, "Application of a continuous wave radar for human gait recognition," in Proceedings of the Signal Processing, Sensor Fusion, and Target Recognition XIV, pp. 538-548, Orlando, Fla, USA, March 2005.

[19] A. Lin and H. Ling, "Through-wall measurements of a Doppler and Direction-of-Arrival (DDOA) radar for tracking indoor movers," in Proceedings of the IEEE Antennas and Propagation Society International Symposium and USNC/URSI Meeting, vol. 3B, pp. 322-325, July 2005.

[20] R. Tan and R. Bender, "Analysis of Doppler Measurements of People," in Proceedings of SPIE Targets and Backgrounds XII: Characterization and Representation, R. Wendell Watkins and Dieter Clement, Eds., vol. 6239, pp. 623908-623901, 2006.

[21] I. Bilik, J. Tabrikian, and A. Cohen, "GMM-based target classification for ground surveillance Doppler radar," IEEE 
Transactions on Aerospace and Electronic Systems, vol. 42, no. 1, pp. 267-278, 2006.

[22] J. Silvious, J. Clark, T. Pizzillo, and D. Tahmoush, "Microdoppler phenomenology of humans at UHF and Ku-band for biometric characterization," in Proceedings of the Radar Sensor Technology XIII, Orlando, Fla, USA, April 2009.

[23] S. Björklund, H. Petersson, A. Nezirovic, M. B. Guldogan, and F. Gustafsson, "Millimeter-wave radar micro-Doppler signatures of human motion," in Proceedings of the International Radar Symposium, (IRS '11), pp. 167-174, September 2011.

[24] Y. Wang and A. E. Fathy, "Micro-Doppler signatures for intelligent human gait recognition using a UWB impulse radar," in Proceedings of the IEEE International Symposium on Antennas and Propagation and USNC/URSI National Radio Science Meeting (APSURSI '11), pp. 2103-2106, Spokane, Wash, USA, July 2011.

[25] O. R. Fogle and B. D. Rigling, "Micro-range/micro-Doppler decomposition of human radar signatures," IEEE Transactions on Aerospace and Electronic Systems, vol. 48, no. 4, pp. 30583072, 2012.

[26] N. F. Troje, "Decomposing biological motion: a framework for analysis and synthesis of human gait patterns," Journal of Vision, vol. 2, no. 5, pp. 371-387, 2002.

[27] T. Dogaru, C. Le, and G. Kirose, "Time-Frequency Analysis of a Moving Human Doppler Signature,” ARL-TR-4728, 2009.

[28] D. Tahmoush, R. Wellman, and J. Silvious, "A compact persistent surveillance radar with micro-doppler capabilities," in Proceedings of the MSS National Symposium, Las Vegas, Nev, USA, August 2009.

[29] A. Freeman, "Fitting a two-component scattering model to polarimetric SAR data from forests," IEEE Transactions on Geoscience and Remote Sensing, vol. 45, no. 8, pp. 2583-2592, 2007. 

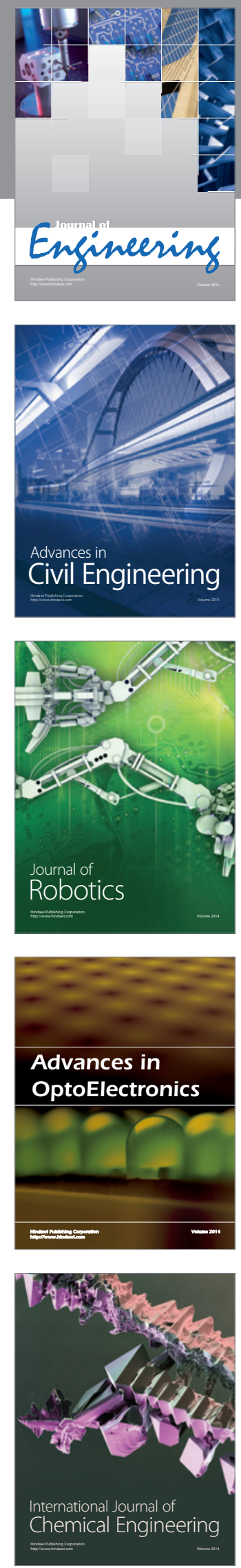

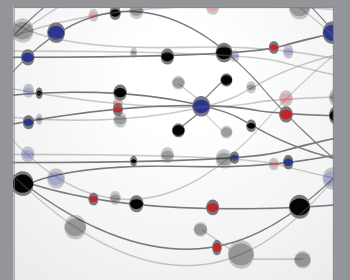

The Scientific World Journal
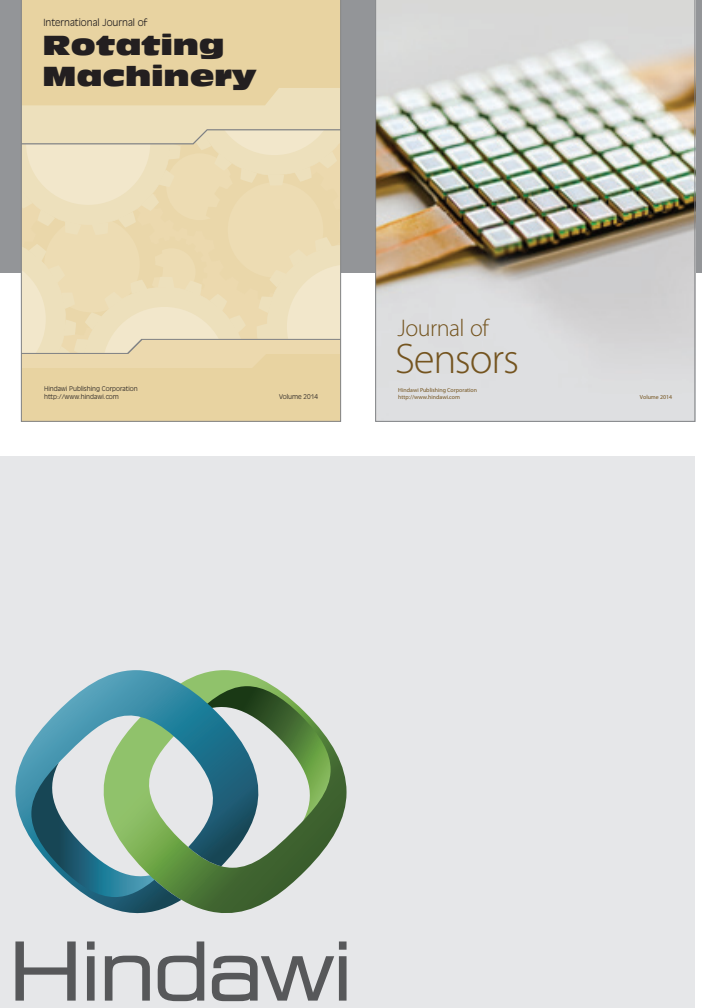

Submit your manuscripts at http://www.hindawi.com
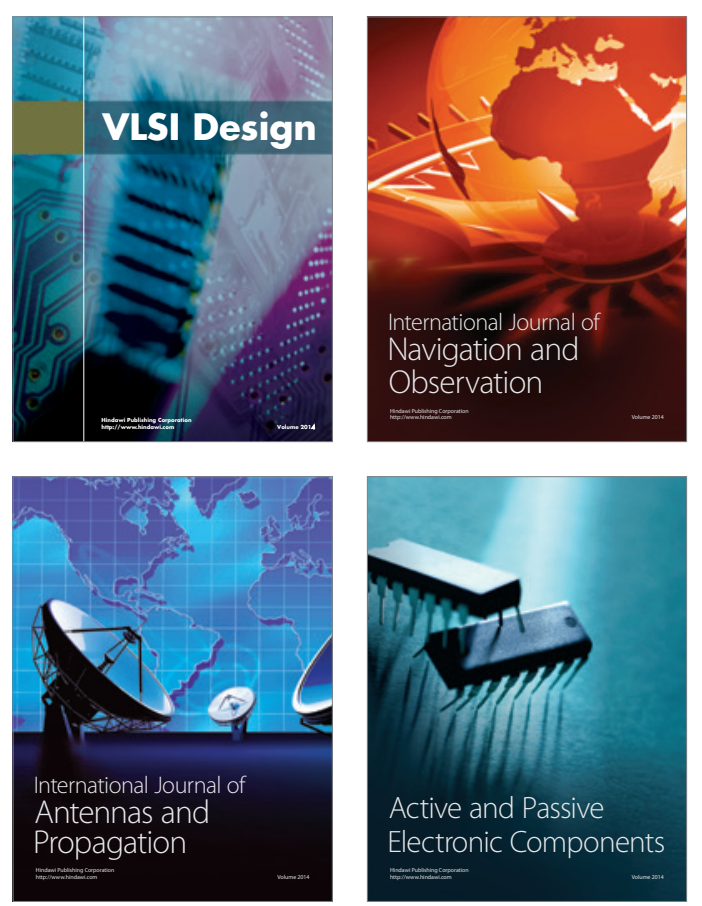
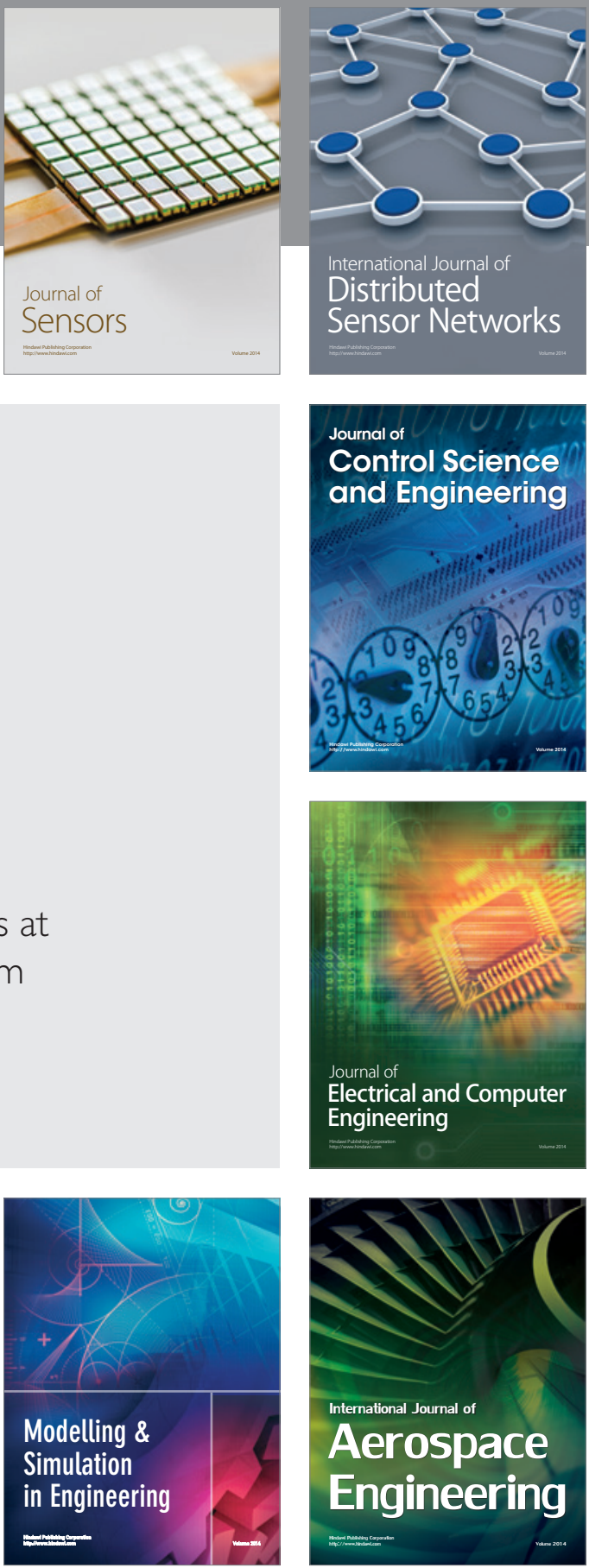

Journal of

Control Science

and Engineering
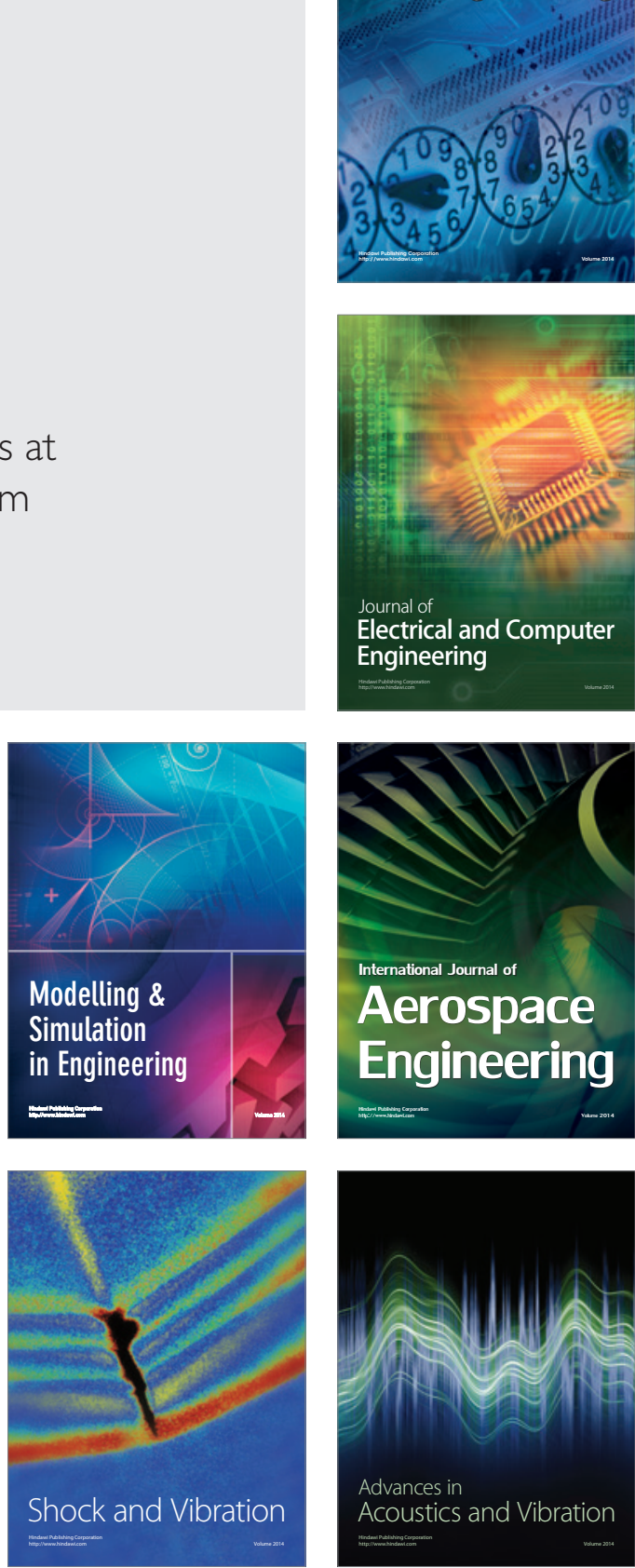